\title{
Reactivity of Pyridine Dipyrrolide Iron(II) Complexes with Organic Azides: C-H Amination and Iron Tetrazene Formation
}

Brett M. Hakey ${ }^{\dagger}$, Jonathan M. Darmon ${ }^{\ddagger}$, Novruz G. Akhmedov ${ }^{\dagger}$, Jeffrey L.

$$
\text { Petersen }^{\dagger}, \text { Carsten Milsmann }{ }^{\dagger *}
$$

${ }^{\dagger} \mathrm{C}$. Eugene Bennett Department of Chemistry, West Virginia University, 100 Prospect Street Morgantown, WV, USA, 26506.

‡Department of Chemistry, Princeton University, Princeton, New Jersey, USA, 08544. 


\section{Table of Contents}

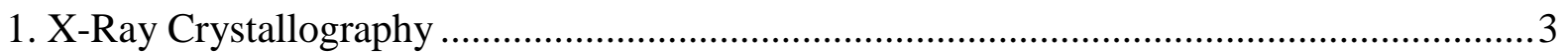

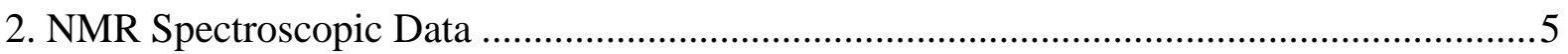

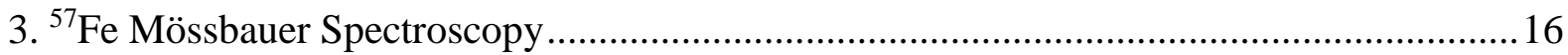

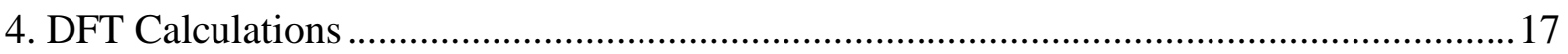

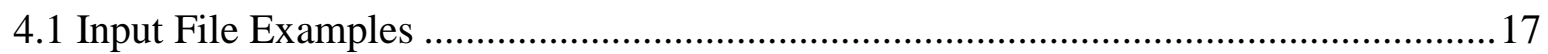

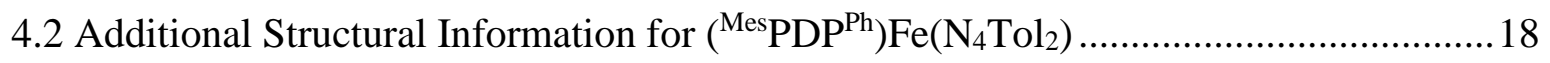

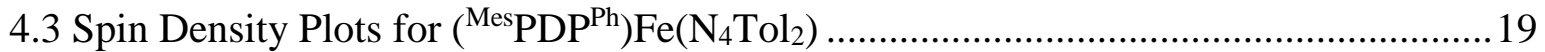




\section{X-Ray Crystallography}

Table S1. Crystallographic Data Collection and Refinement Details for $\left(\mathrm{NH}^{1} \mathrm{Ad}-\right.$ $\left.{ }^{\mathrm{Mes}} \mathrm{PDP}^{\mathrm{Ph}}\right) \mathrm{Fe}($ thf $)$ and $\left({ }^{\mathrm{Mes}} \mathrm{PDP}^{\mathrm{Ph}}\right) \mathrm{Fe}\left(\mathrm{N}_{4} \mathrm{Tol}_{2}\right)$.

\begin{tabular}{|c|c|c|}
\hline & $\begin{array}{c}\left(\mathrm{NH}^{1} \mathrm{Ad}-{ }^{\mathrm{Mes}} \mathrm{PDP}^{\mathrm{Ph}}\right) \mathrm{Fe}(\text { thf }) \\
\cdot 2 \mathrm{THF} \cdot 1 / 2 \mathrm{C}_{5} \mathrm{H}_{12}\end{array}$ & $\begin{array}{c}\left(\mathrm{C}_{43} \mathrm{H}_{37} \mathrm{~N}_{3}\right) \mathrm{Fe}\left(\mathrm{N}_{4} \mathrm{Tol}_{2}\right) \\
\cdot 1 / 2 \mathrm{C}_{7} \mathrm{H}_{8}\end{array}$ \\
\hline chem. formula & $\mathrm{C}_{67.50} \mathrm{H}_{82} \mathrm{FeN}_{4} \mathrm{O}_{3}$ & $\mathrm{C}_{60.50} \mathrm{H}_{55} \mathrm{FeN}_{7}$ \\
\hline cryst size, $\mathrm{mm}^{3}$ & $0.136 \times 0.261 \times 0.571$ & $0.094 \times 0.109 \times 0.399$ \\
\hline Fw g/mol & 1053.22 & 935.96 \\
\hline space group & $\begin{array}{c}\text { P } 2{ }_{1} / \text { n (non-standard setting of } \\
\left.2_{1} / \mathrm{c}, \text { No. } 14\right)\end{array}$ & P-1 (No. 2) \\
\hline $\mathrm{a}, \AA$ & $18.1621(8)$ & $12.5577(8)$ \\
\hline $\mathrm{b}, \AA$ & $12.4351(6)$ & $14.3176(9)$ \\
\hline $\mathrm{c}, \AA$ & $26.2424(11)$ & $14.8373(9)$ \\
\hline$\alpha, \operatorname{deg}$ & 90 & $112.3292(15)$ \\
\hline$\beta$, deg & $102.6579(13)$ & $96.6713(17)$ \\
\hline$\gamma, \operatorname{deg}$ & 90 & $98.2021(16)$ \\
\hline $\mathrm{V}, \AA$ & $5782.7(4)$ & $2399.8(3)$ \\
\hline Z & 4 & 2 \\
\hline $\mathrm{T}, \mathrm{K}$ & $100(2)$ & $105(2)$ \\
\hline$\rho$ calcd, $\mathrm{g} \mathrm{cm}^{-3}$ & 1.210 & 1.295 \\
\hline reflns collected $/ 2 \Theta_{\max }$ & $121272 / 55.16$ & $46269 / 50.20$ \\
\hline unique reflns/I $>2 \sigma(I)$ & $13356 / 9982$ & $8485 / 5870$ \\
\hline No. of params/restraints & $644 / 5$ & $646 / 0$ \\
\hline$\lambda, \AA$ & 0.71073 & 0.71073 \\
\hline $\mathrm{R} 1$ a/goodness of fit ${ }^{\mathrm{b}}$ & $0.0521 / 1.016$ & $0.0619 / 1.014$ \\
\hline $\mathrm{wR}^{2}{ }^{\mathrm{c}}(I>2 \sigma(I))$ & 0.1199 & 0.1079 \\
\hline Residual density, e $\AA^{-3}$ & 0.554 and -0.468 & 0.600 and -0.832 \\
\hline
\end{tabular}


Table S2. Crystallographic Data Collection and Refinement Details for Fe $\left(\{\mathrm{NHBn}\} 2_{2}{ }^{\mathrm{Mes}} \mathrm{PDP}^{\mathrm{Ph}}\right)$ and $\left({ }^{\mathrm{Mes} P D P}{ }^{\mathrm{Ph}}\right) \mathrm{Fe}\left(\mathrm{N}_{4} \mathrm{Bn}_{2}\right)(\mathrm{HN}=\mathrm{CHPh})$.

\begin{tabular}{|c|c|c|}
\hline & $\mathrm{Fe}\left(\{\mathrm{NHBn}\}_{2}{ }^{\mathrm{Mes}} \mathrm{PDP}^{\mathrm{Ph}}\right)$ & $\left({ }^{\mathrm{Mes}} \mathrm{PDP}^{\mathrm{Ph}}\right) \mathrm{Fe}\left(\mathrm{N}_{4} \mathrm{Bn}_{2}\right)(\mathrm{HN}=\mathrm{CHPh})$ \\
\hline chem. formula & $\mathrm{C}_{57} \mathrm{H}_{51} \mathrm{~N}_{5} \mathrm{Fe}$ & $\mathrm{C}_{71} \mathrm{H}_{66} \mathrm{FeN}_{8}$ \\
\hline cryst size, $\mathrm{mm}^{3}$ & $0.148 \times 0.551 \times 0.725$ & $0.126 \times 0.330 \times 0.745$ \\
\hline Fw g/mol & 861.87 & 1087.16 \\
\hline space group & C $2 / \mathrm{c}($ No. 15$)$ & $P(-1)($ No. 2) \\
\hline $\mathrm{a}, \AA$ & $20.7165(9)$ & $11.5957(6)$ \\
\hline $\mathrm{b}, \AA$ & $25.3670(11)$ & $11.6538(5)$ \\
\hline $\mathrm{c}, \AA$ & $8.8099(4)$ & $23.2291(11)$ \\
\hline$\alpha, \operatorname{deg}$ & 90 & $86.3130(14)$ \\
\hline$\beta$, deg & $101.5215(14)$ & $81.8786(15)$ \\
\hline$\gamma, \operatorname{deg}$ & 90 & $66.5993(14)$ \\
\hline $\mathrm{V}, \AA$ & $4536.4(3)$ & $2851.8(2)$ \\
\hline $\mathrm{Z}$ & 4 & 2 \\
\hline $\mathrm{T}, \mathrm{K}$ & $100(2)$ & $100(2)$ \\
\hline$\rho$ calcd, $\mathrm{g} \mathrm{cm}^{-3}$ & 1.262 & 1.266 \\
\hline reflns collected $/ 2 \Theta_{\max }$ & $55683 / 65.32$ & $63800 / 60.22$ \\
\hline unique reflns/I $>2 \sigma(I)$ & $8309 / 6724$ & $16669 / 13075$ \\
\hline No. of params/restraints & $292 / 0$ & $668 / 0$ \\
\hline$\lambda, \AA$ & 0.71073 & 0.71073 \\
\hline $\mathrm{R} 1^{\mathrm{a}}$ /goodness of fit ${ }^{\mathrm{b}}$ & $0.0498 / 1.062$ & $0.0516 / 1.027$ \\
\hline $\mathrm{wR}^{\mathrm{c}}(I>2 \sigma(I))$ & 0.1182 & 0.1133 \\
\hline Residual density, e $\AA^{-3}$ & 0.654 and -0.336 & 0.684 and -0.609 \\
\hline
\end{tabular}


2. NMR Spectroscopic Data

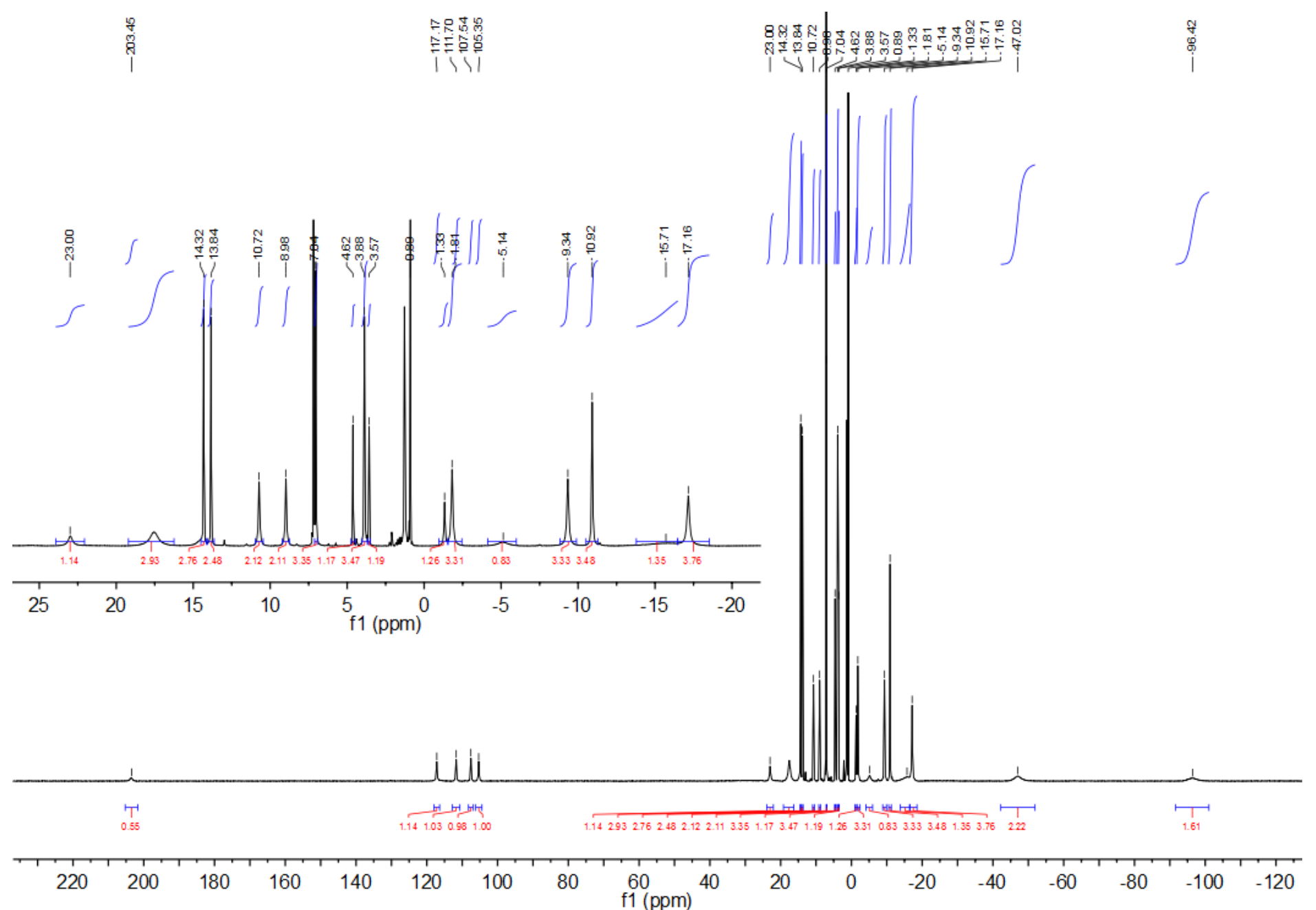

Figure S1. ${ }^{1} \mathrm{H}$ NMR spectrum of $\mathrm{Fe}\left(\mathrm{NH}^{1} \mathrm{Ad}-{ }^{\mathrm{Mes}} \mathrm{PDP}^{\mathrm{Ph}}\right)$ in benzene- $d_{6}$. 


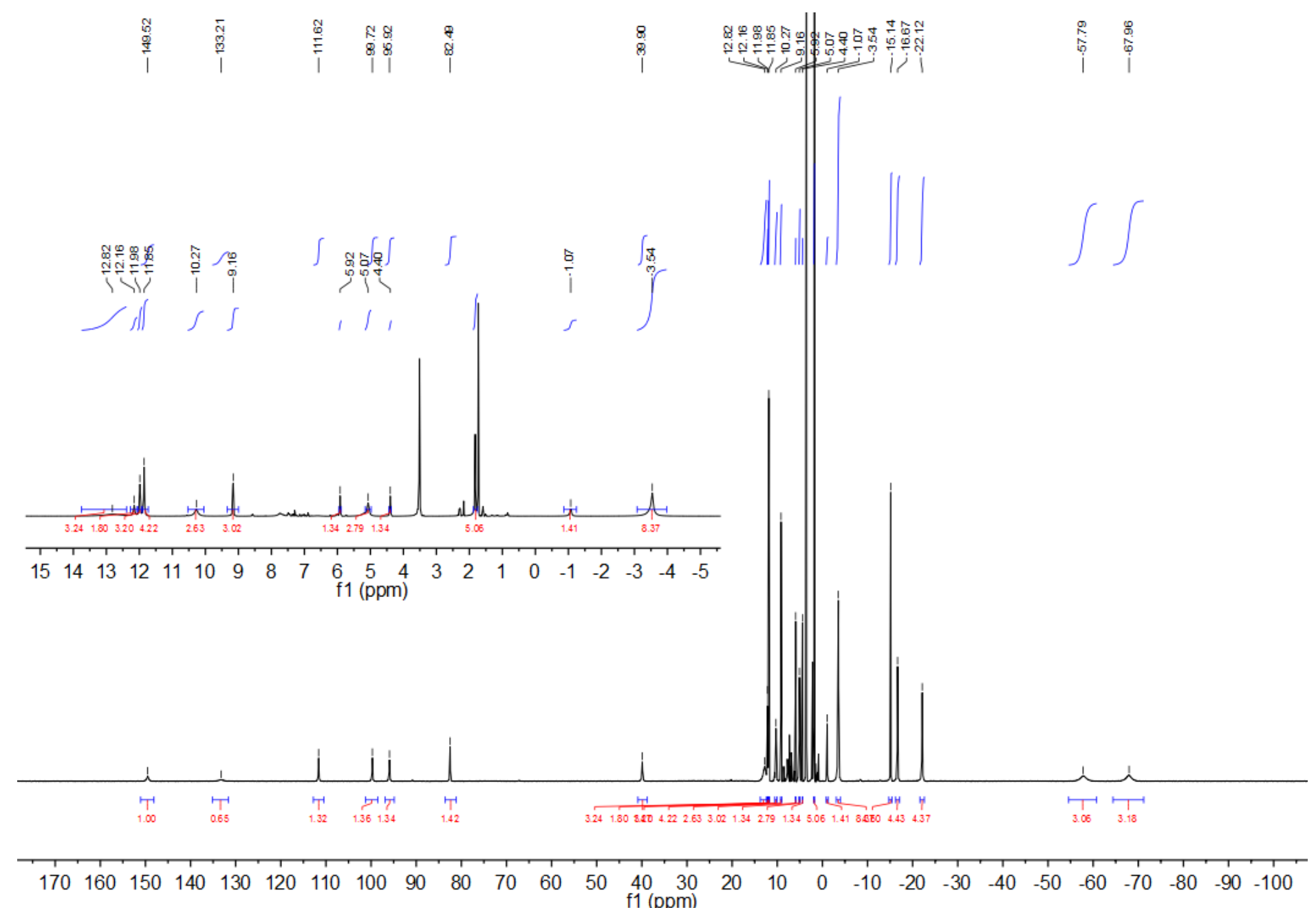

Figure S2. ${ }^{1} \mathrm{H}$ NMR spectrum of $\left(\mathrm{NH}^{1} \mathrm{Ad}-{ }^{\mathrm{Mes}} \mathrm{PDP}{ }^{\mathrm{Ph}}\right) \mathrm{Fe}($ thf $)$ in THF- $d_{8}$. 


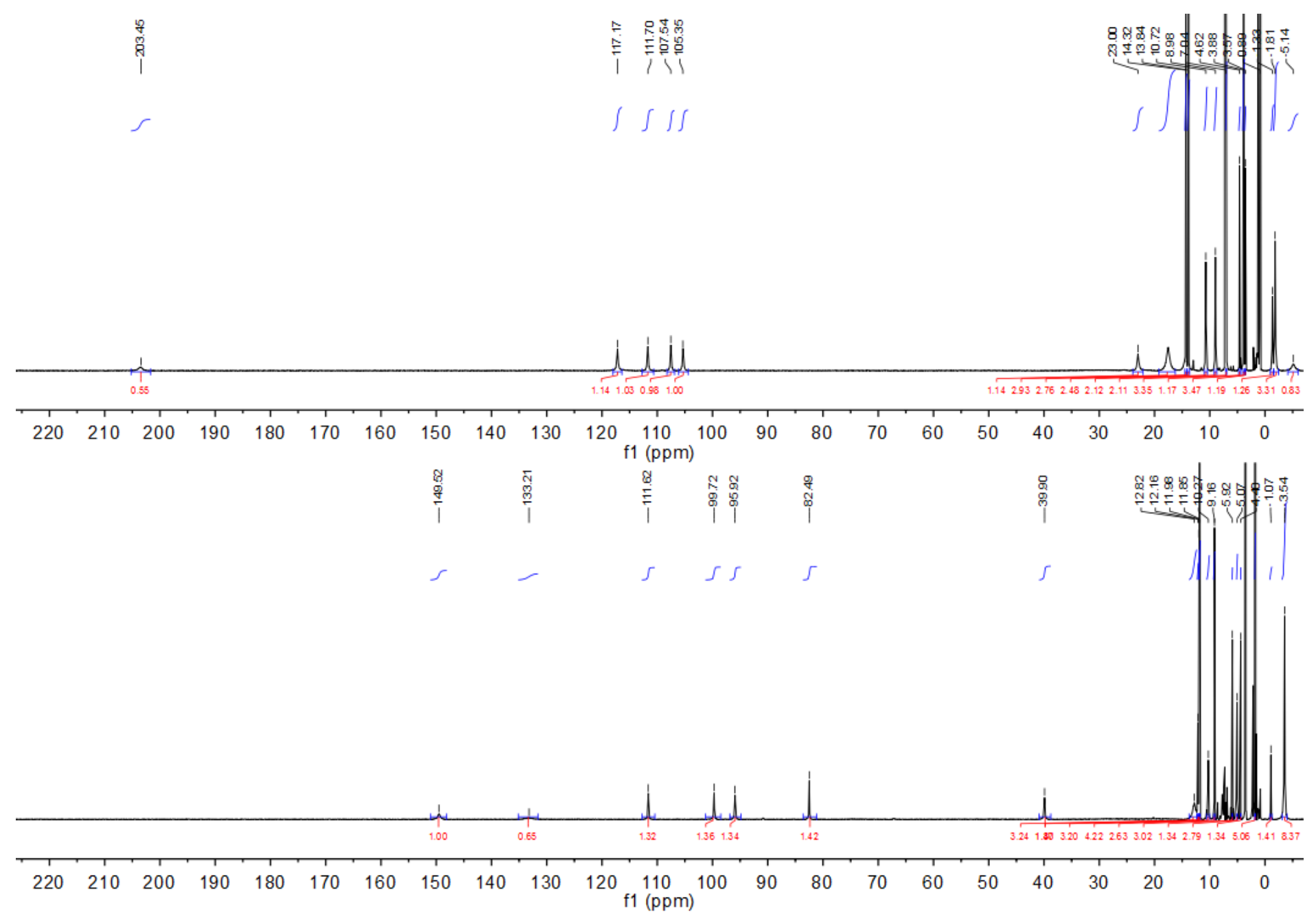

Figure S3. Top: ${ }^{1} \mathrm{H}$ NMR spectrum of $\mathrm{Fe}\left(\mathrm{NH}^{1} \mathrm{Ad}-{ }^{\mathrm{Mes}} \mathrm{PDP}^{\mathrm{Ph}}\right)$ in benzene- $d_{6}$. Bottom: ${ }^{1} \mathrm{H}$ NMR spectrum of $\left(\mathrm{NH}^{1} \mathrm{Ad}-{ }^{\mathrm{Mes}} \mathrm{PDP} \mathrm{Ph}^{\mathrm{Ph}}\right) \mathrm{Fe}(\mathrm{thf}) \mathrm{in}_{\mathrm{THF}} d_{8}$. 

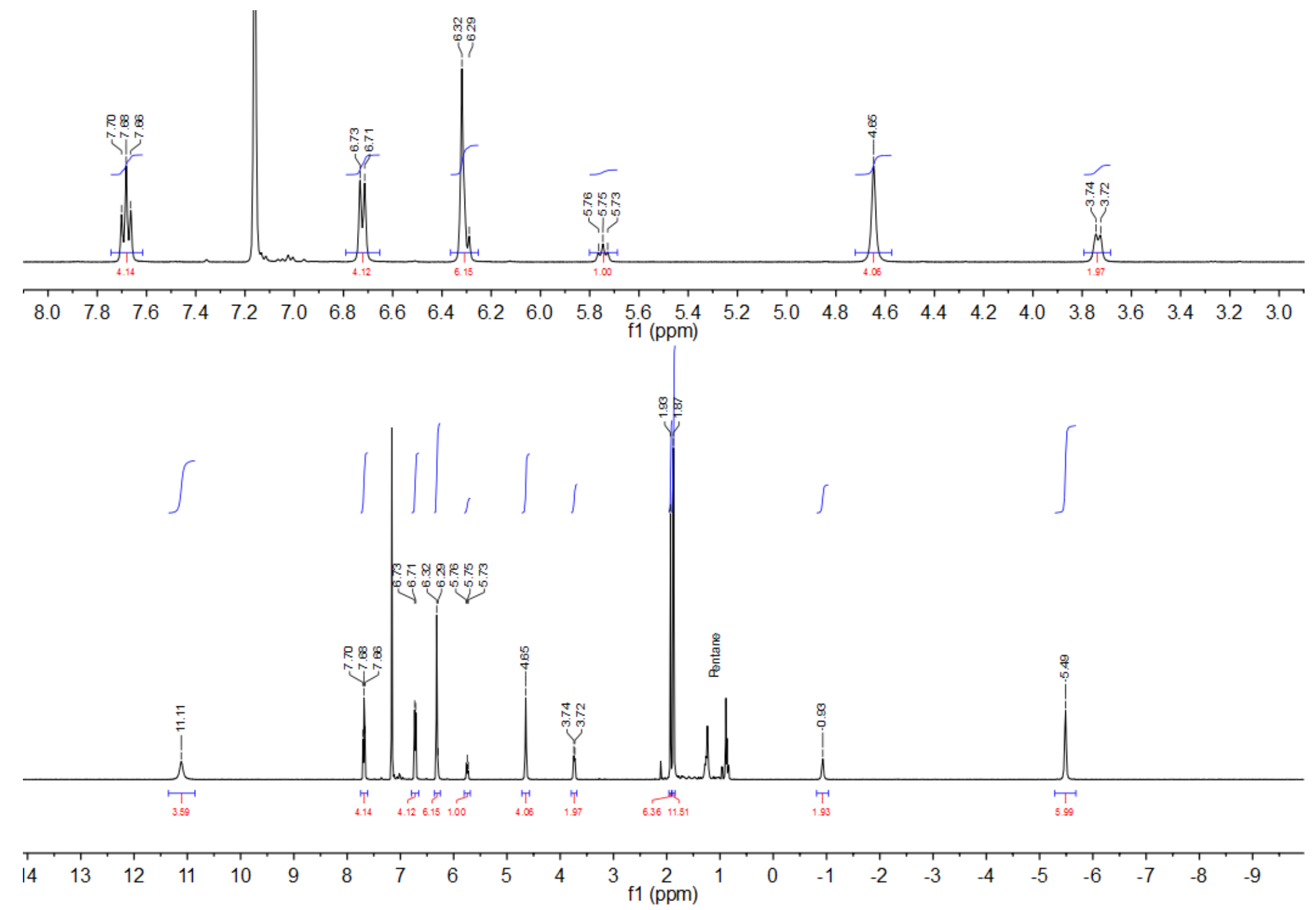

Figure S4. ${ }^{1} \mathrm{H}$ NMR spectrum of $\left({ }^{\mathrm{Mes}} \mathrm{PDP}^{\mathrm{Ph}}\right) \mathrm{Fe}\left(\mathrm{N}_{4} \mathrm{Tol}_{2}\right)$ in benzene- $d_{6}$. 


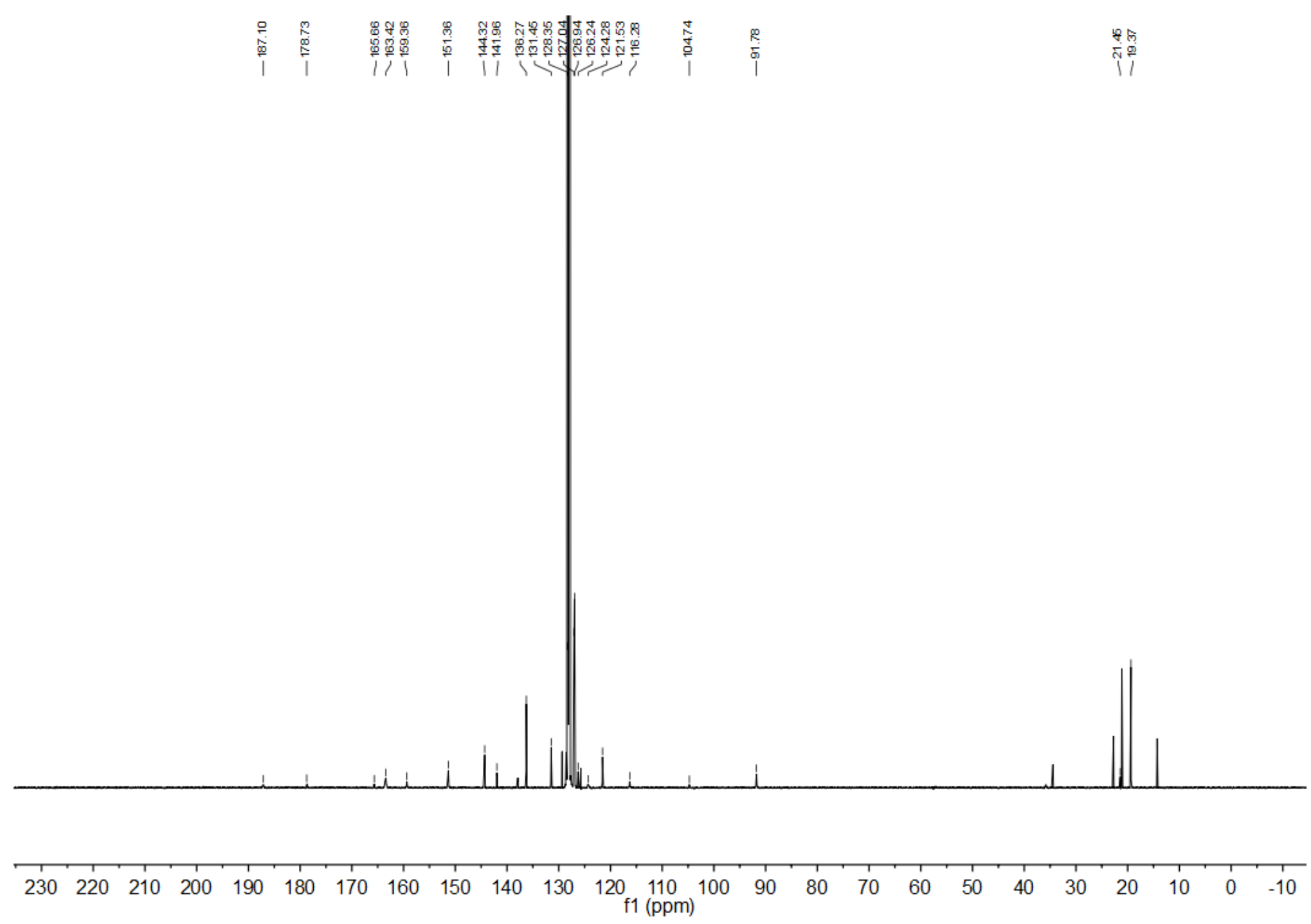

Figure S5. ${ }^{13} \mathrm{C}$ NMR spectrum of $\left({ }^{\mathrm{Mes}} \mathrm{PDP}^{\mathrm{Ph}}\right) \mathrm{Fe}\left(\mathrm{N}_{4} \mathrm{Tol}_{2}\right)$ in benzene- $d_{6}$. 


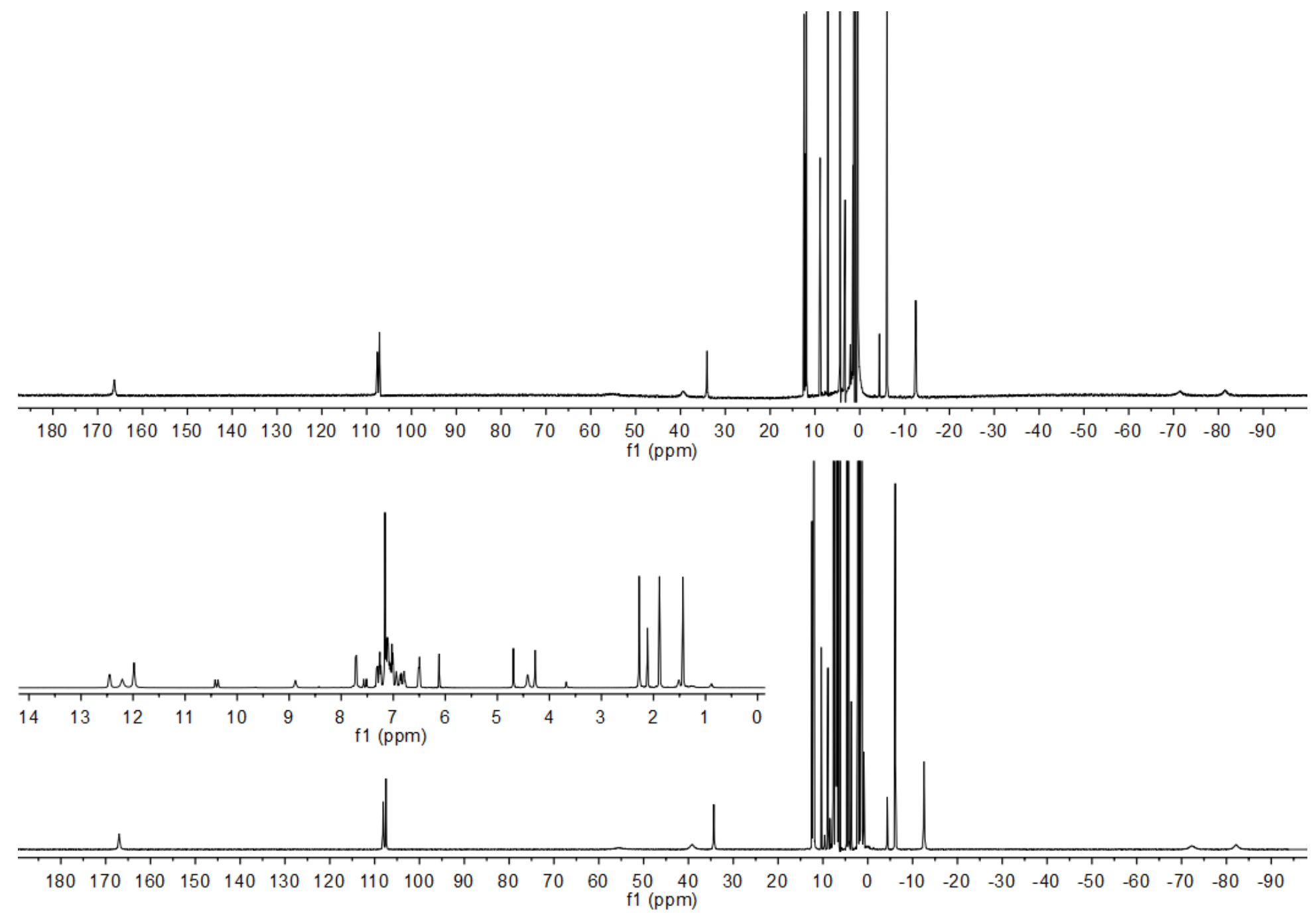

Figure S6. Top: ${ }^{1} \mathrm{H}$ NMR spectrum of isolated crystalline sample of $\mathrm{Fe}\left(\{\mathrm{NHBn}\} 2_{2}-{ }^{\mathrm{Mes}} \mathrm{PDP}{ }^{\mathrm{Ph}}\right)$ in benzene- $d_{6}$. Bottom: ${ }^{1} \mathrm{H}$ NMR spectrum of crude mixture of $\mathrm{Fe}\left(\{\mathrm{NHBn}\}_{2}{ }^{\mathrm{Mes}} \mathrm{PDP}^{\mathrm{Ph}}\right)$ and $\left({ }^{\mathrm{Mes}} \mathrm{PDP}{ }^{\mathrm{Ph}}\right) \mathrm{Fe}\left(\mathrm{N}_{4} \mathrm{Bn}_{2}\right)(\mathrm{HN}=\mathrm{CHPh})$ in benzene- $d_{6}$. 


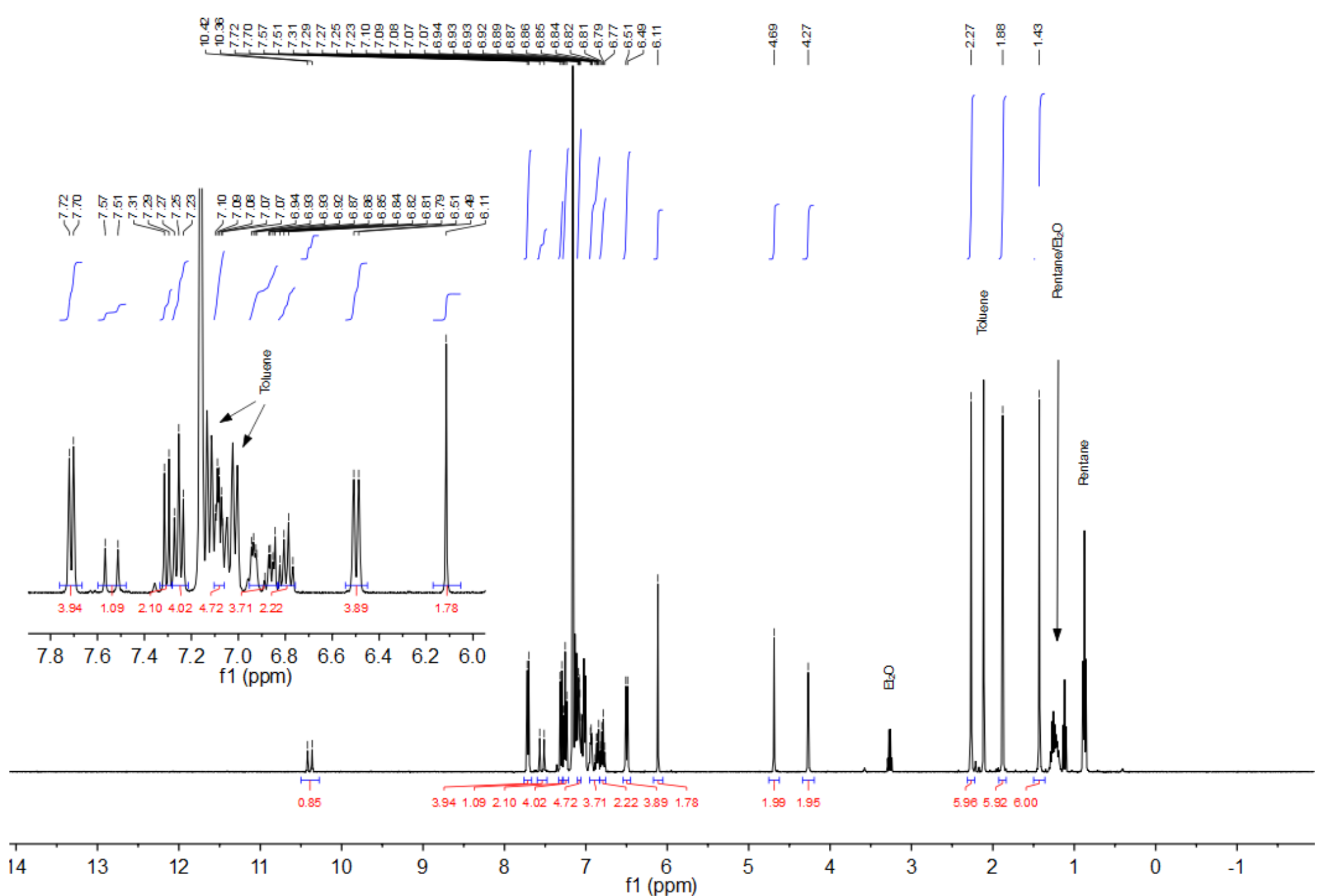

Figure S7. ${ }^{1} \mathrm{H}$ NMR spectrum of $\left({ }^{\mathrm{Mes}} \mathrm{PDP}^{\mathrm{Ph}}\right) \mathrm{Fe}\left(\mathrm{N}_{4} \mathrm{Bn}_{2}\right)(\mathrm{HN}=\mathrm{CHPh})$ in benzene- $d_{6}$. 


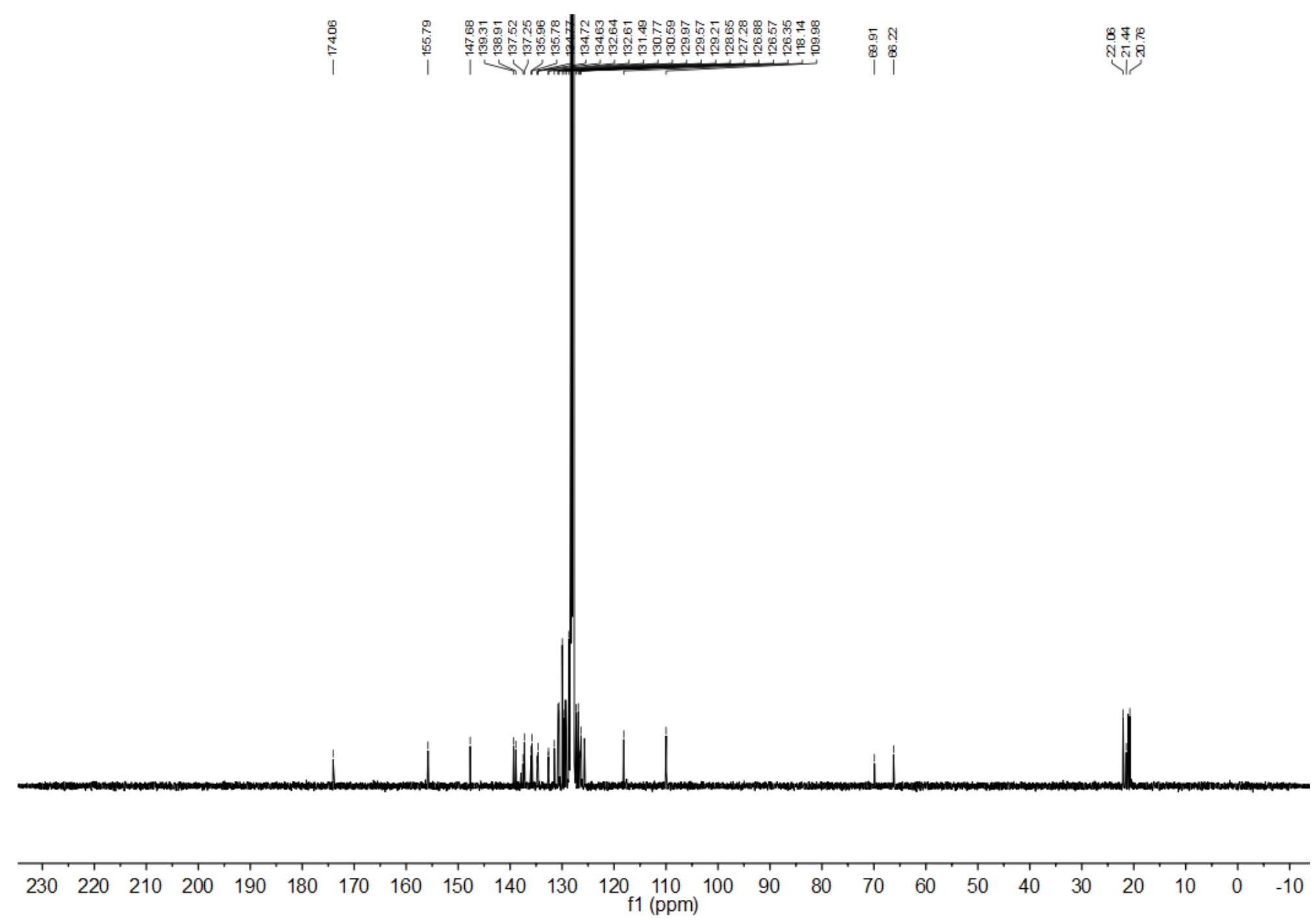

Figure S8. ${ }^{13} \mathrm{C}$ NMR spectrum $\left({ }^{\mathrm{Mes}} \mathrm{PDP}^{\mathrm{Ph}}\right) \mathrm{Fe}\left(\mathrm{N}_{4} \mathrm{Bn}_{2}\right)(\mathrm{HN}=\mathrm{CHPh})$ in benzene- $d_{6}$. 


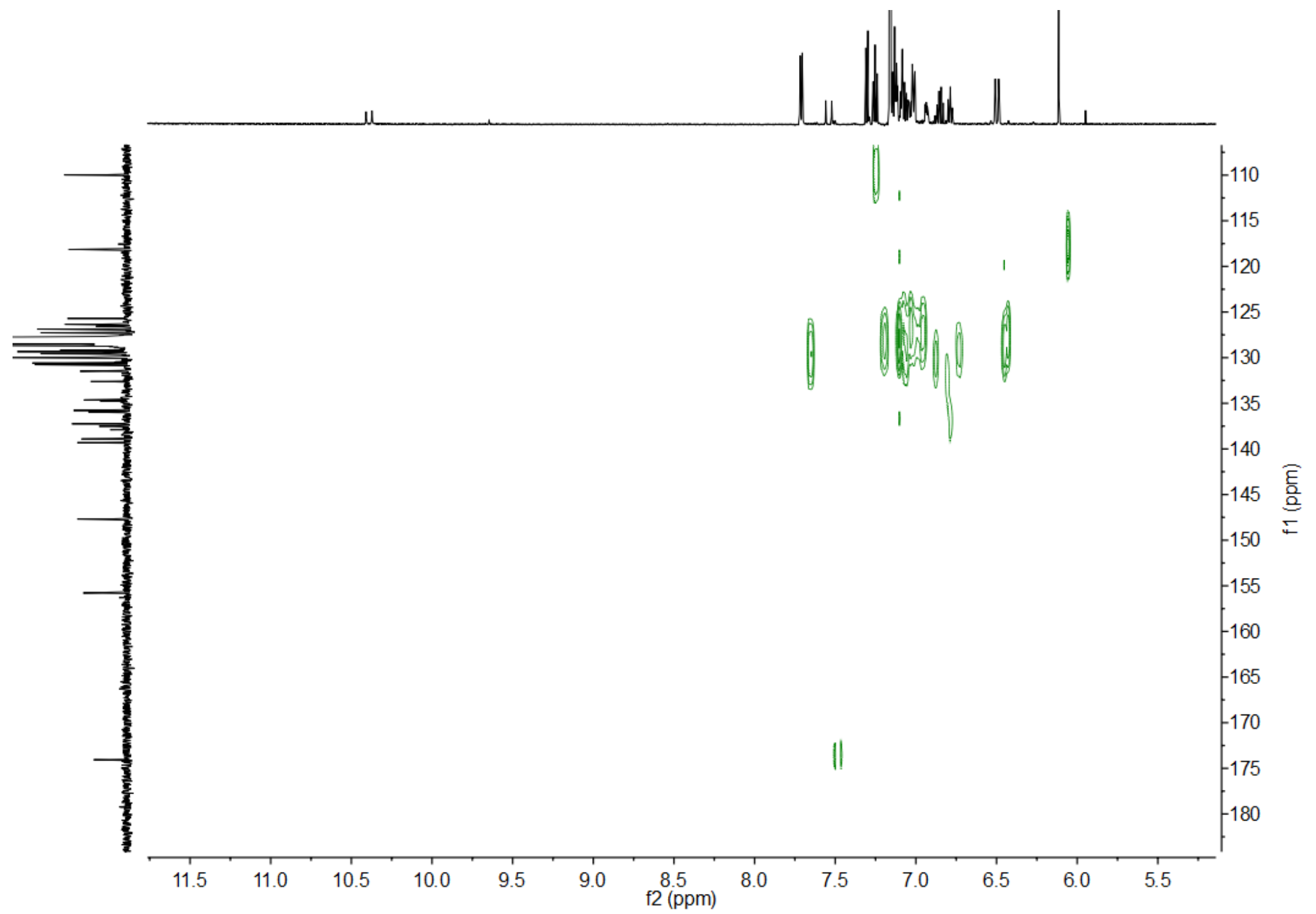

Figure S9. HSQC spectrum of $\left({ }^{\mathrm{Mes}} \mathrm{PDP}^{\mathrm{Ph}}\right) \mathrm{Fe}\left(\mathrm{N}_{4} \mathrm{Bn}_{2}\right)(\mathrm{HN}=\mathrm{CHPh})$ in benzene- $d_{6}$ indicating aldimine $\mathrm{C} H$ correlation. 


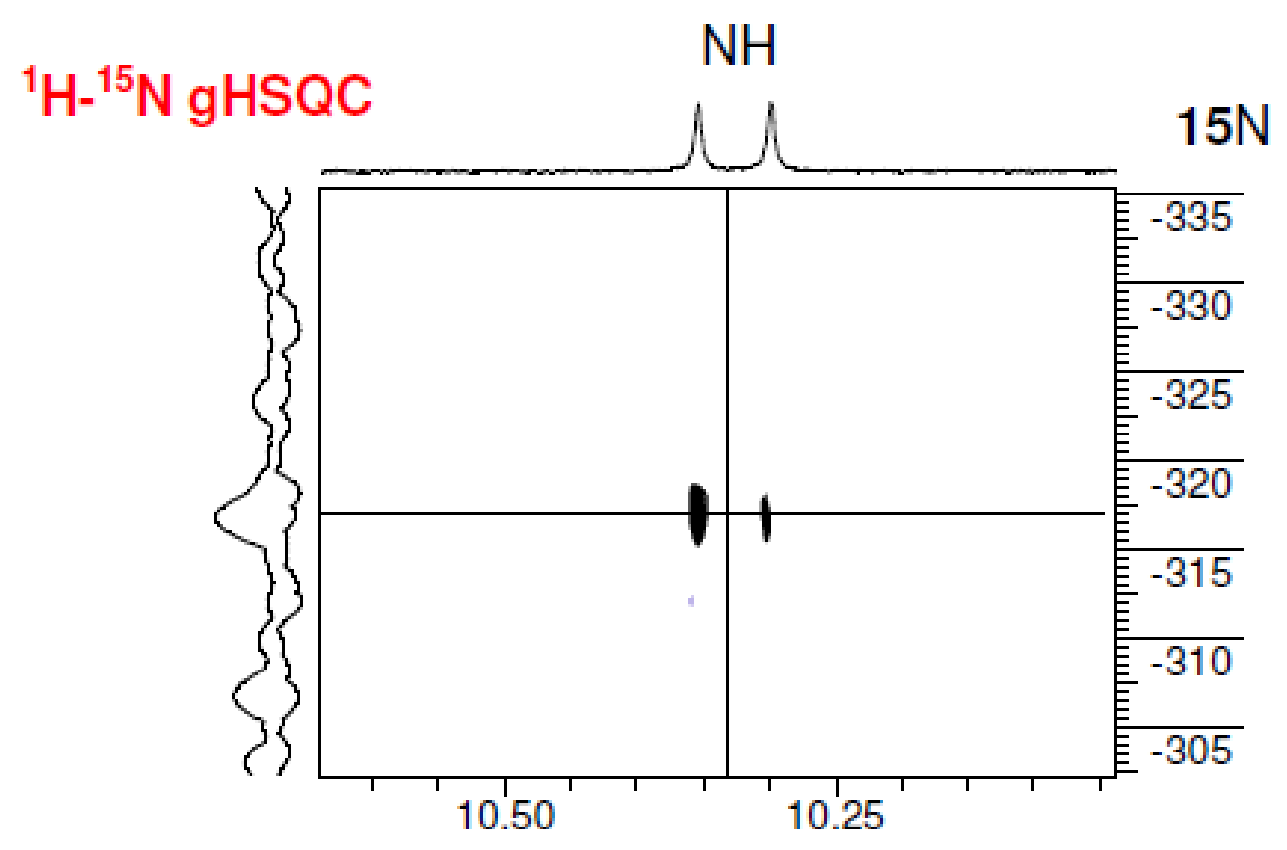

Figure S10. ${ }^{1} \mathrm{H}_{-}{ }^{15} \mathrm{~N}$ HSQC spectrum of $\left({ }^{\mathrm{Mes}} \mathrm{PDP}^{\mathrm{Ph}}\right) \mathrm{Fe}\left(\mathrm{N}_{4} \mathrm{Bn} 2\right)(\mathrm{HN}=\mathrm{CHPh})$ in benzene- $d_{6}$ indicating aldimine $\mathrm{N} H$ correlation. 


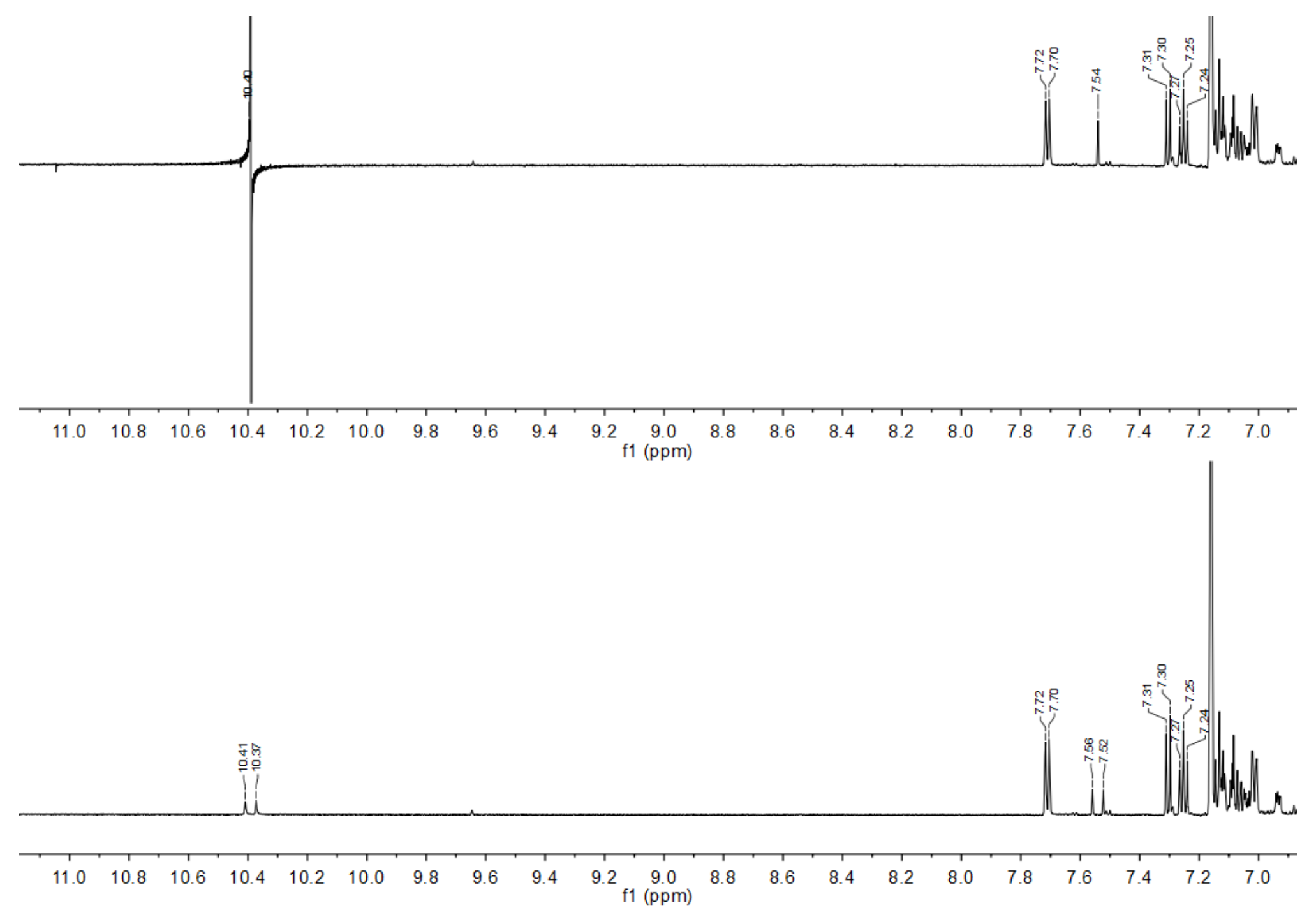

Figure S11. Selective ${ }^{1} \mathrm{H}$ decoupling experiment in benzene- $d_{6}$ indicating aldimine $\mathrm{C} H$ and $\mathrm{N} H$ correlation. 


\section{3. ${ }^{57} \mathrm{Fe}$ Mössbauer Spectroscopy}

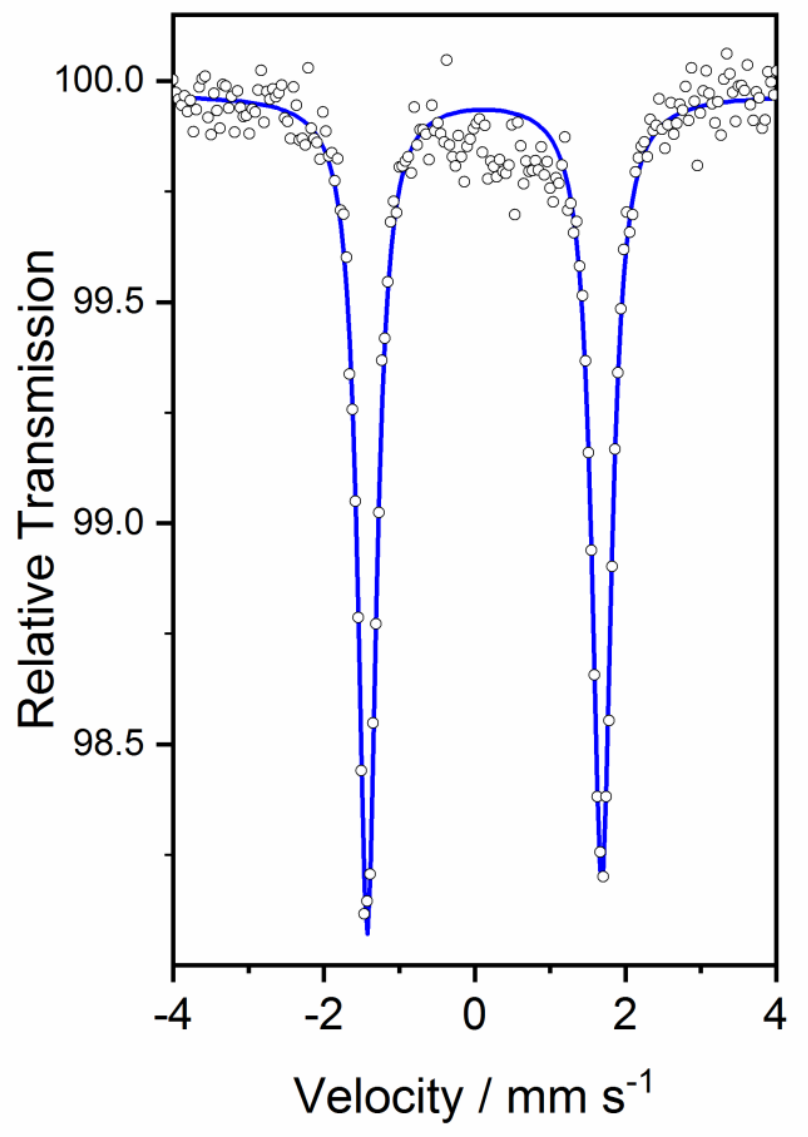

Figure S12. Zero-field Mössbauer spectrum of $\left({ }^{\mathrm{Mes}} \mathrm{PDP}^{\mathrm{Ph}}\right) \mathrm{Fe}\left(\mathrm{N}_{4} \mathrm{Tol}_{2}\right)$ at $80 \mathrm{~K}$. $\delta=0.12 \mathrm{~mm} / \mathrm{s},\left|\Delta \mathrm{E}_{\mathrm{Q}}\right|=3.12 \mathrm{~mm} / \mathrm{s}$. 


\section{DFT Calculations}

\subsection{Input File Examples}

\section{Geometry Optimization}

!UKS B3LYP RIJCOSX tightscf slowconv def2-SVP def2-SVP/J normalprint opt pal8

\%basis newgto 26 "def2-tzvp(-f)" end

newgto 8 "def2-tzvp(-f)" end

newgto 7 "def2-tzvp(-f)" end

newauxgto 26 "def2-tzvp/j" end

newauxgto 8 "def2-tzvp/j" end

newauxgto 7 "def2-tzvp/j" end

end

$\%$ scf maxiter 500

tole $1 \mathrm{e}-7$

tolerr $1 \mathrm{e}-6$

end

*xyz charge multiplicity

$\mathrm{xyz}$ coordinates from $\mathrm{X}$-ray structure

$*$

${ }^{57}$ Mössbauer Quadrupole Splitting $\left(\Delta \mathrm{E}_{\mathrm{Q}}\right)$ and Isomer Shift $(\delta)$

!UKS B3LYP tightscf slowconv def2-SVP normalprint Direct Grid4 FinalGrid5 pal8

\%basis NewGTO 26 "CP(PPP)" end

NewGTO 8 "def2-TZVP(-f)" end

NewGTO 7 "def2-TZVP(-f)" end

end

\%method SpecialGridAtoms 26

SpecialGridIntAcc 7

end

$\%$ scf maxiter 500

tole $1 \mathrm{e}-7$

tolerr 1e-6

end 
*xyz charge multiplicity

xyz coordinates from geometry optimization

$*$

\%eprnmr nuclei $=$ all Fe $\{$ rho, fgrad $\}$

end

\subsection{Additional Structural Information for $\left({ }^{\mathrm{Mes}} \mathrm{PDP}{ }^{\mathrm{Ph}}\right) \mathrm{Fe}\left(\mathrm{N}_{4} \mathrm{Tol}_{2}\right)$}

Table S3. Comparison of Selected Experimental and Computational Bond Lengths for $\left({ }^{\mathrm{Mes}} \mathrm{PDP}{ }^{\mathrm{Ph}}\right) \mathrm{Fe}\left(\mathrm{N}_{4} \mathrm{Tol}_{2}\right)$.

\begin{tabular}{lcccccc}
\hline & Exp, & BS(1,1) & RKS & $\begin{array}{c}\text { BS(3,1) } \\
\text { /UKS3 }\end{array}$ & BS(5,1) & UKS5 \\
\hline $\mathrm{Fe}(1)-\mathrm{N}(1)$ & $1.946(3)$ & 1.999 & 2.006 & 2.024 & 2.128 & 2.002 \\
$\mathrm{Fe}(1)-\mathrm{N}(2)$ & $1.943(3)$ & 1.952 & 1.959 & 1.962 & 2.088 & 1.928 \\
$\mathrm{Fe}(1)-\mathrm{N}(3)$ & $1.955(3)$ & 1.998 & 2.005 & 2.016 & 2.130 & 2.005 \\
$\mathrm{Fe}(1)-\mathrm{N}(4)$ & $1.867(3)$ & 1.925 & 1.896 & 1.889 & 2.047 & 1.968 \\
$\mathrm{Fe}(1)-\mathrm{N}(7)$ & $1.803(3)$ & 1.825 & 1.803 & 2.121 & 2.034 & 2.102 \\
$\mathrm{~N}(4)-\mathrm{N}(5)$ & $1.299(4)$ & 1.306 & 1.295 & 1.303 & 1.298 & 1.313 \\
$\mathrm{~N}(5)-\mathrm{N}(6)$ & $1.336(4)$ & 1.313 & 1.329 & 1.319 & 1.323 & 1.297 \\
$\mathrm{~N}(6)-\mathrm{N}(7)$ & $1.318(4)$ & 1.302 & 1.289 & 1.295 & 1.300 & 1.319
\end{tabular}




\subsection{Spin Density Plots for $\left({ }^{\mathrm{Mes}} \mathrm{PDP}{ }^{\mathrm{Ph}}\right) \mathrm{Fe}\left(\mathrm{N}_{4} \mathrm{Tol}_{2}\right)$}

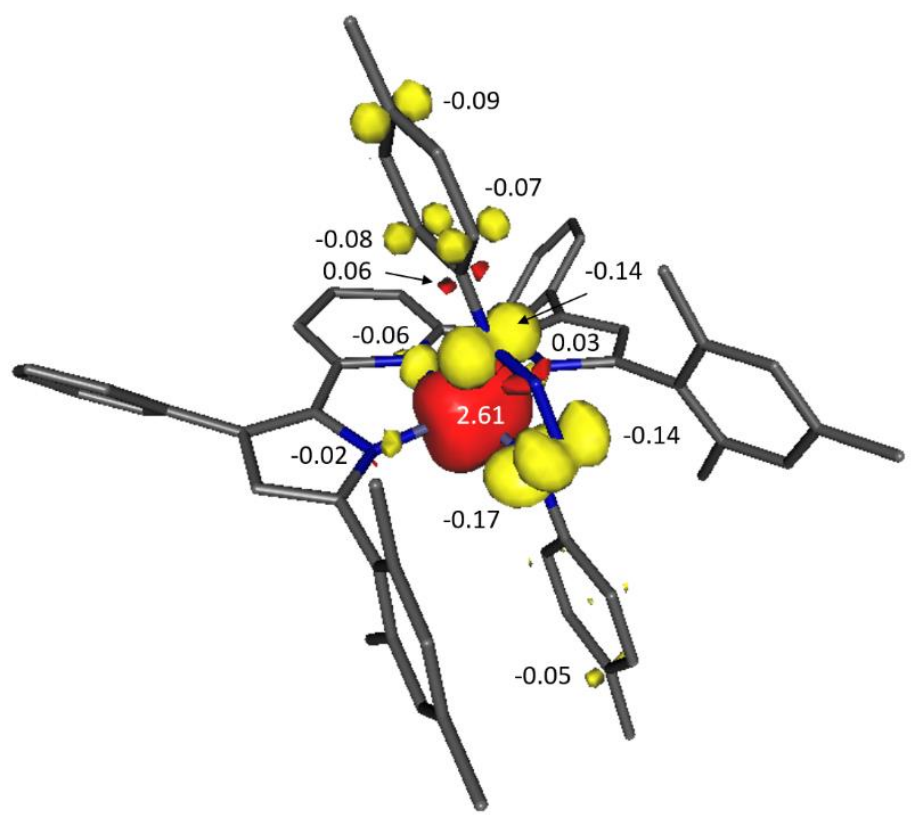

Figure S13. Spin density distributions obtained via Mulliken population analysis for $\left({ }^{\mathrm{Mes}} \mathrm{PDP}^{\mathrm{Ph}}\right) \mathrm{Fe}\left(\mathrm{N}_{4} \mathrm{Tol}_{2}\right)$ using a BS(3,1) approach.

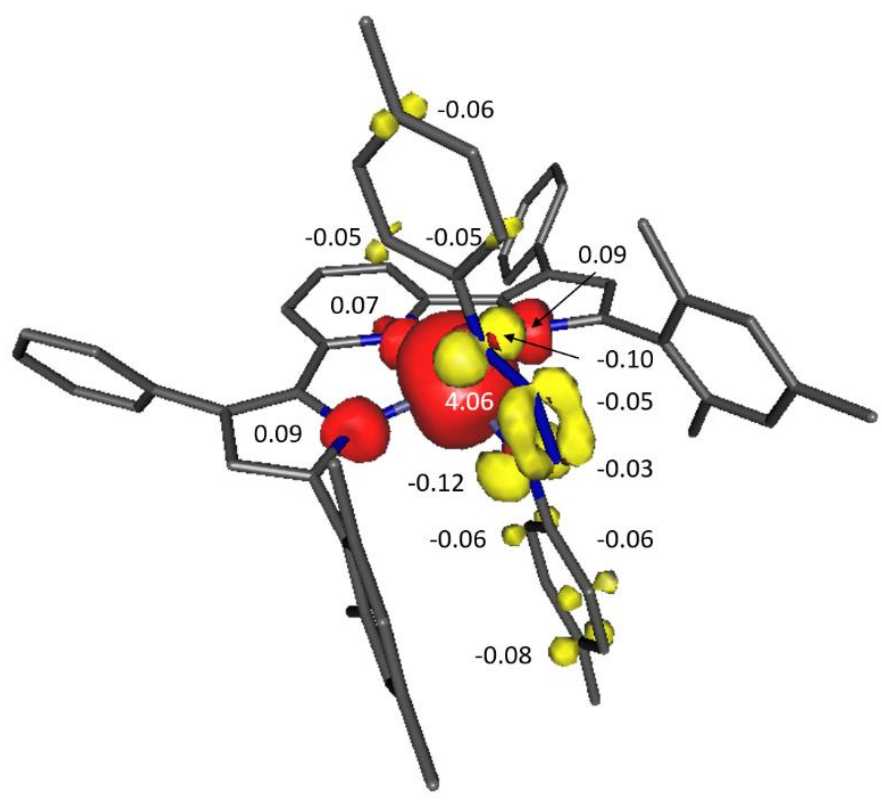

Figure S14. Spin density distributions obtained via Mulliken population analysis for $\left({ }^{\mathrm{Mes}} \mathrm{PDP}{ }^{\mathrm{Ph}}\right) \mathrm{Fe}\left(\mathrm{N}_{4} \mathrm{Tol}_{2}\right)$ using a BS$(5,1)$ approach. 


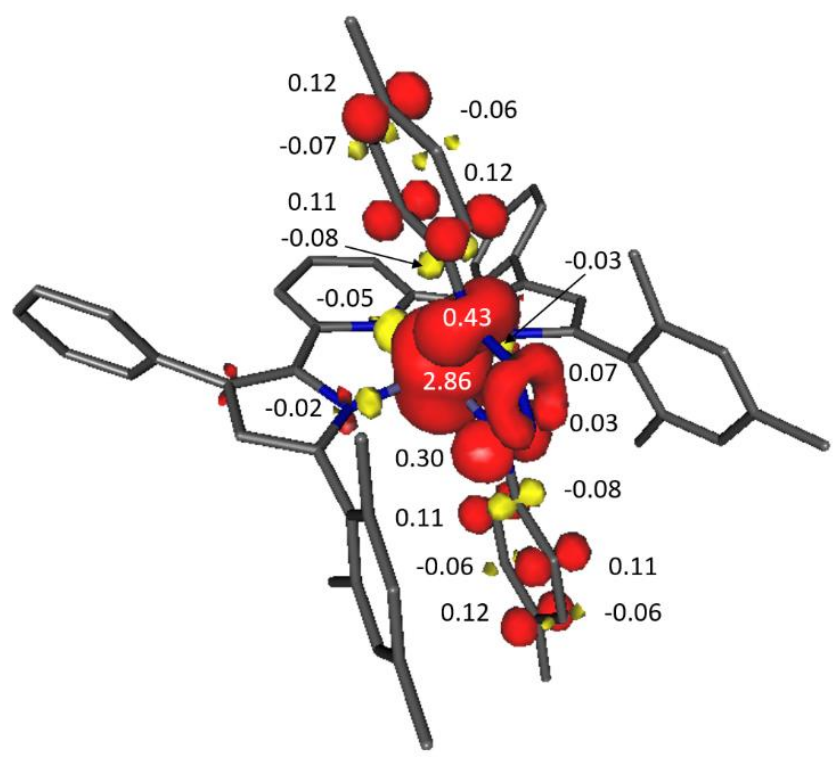

Figure S15. Spin density distributions obtained via Mulliken population analysis for $\left({ }^{\mathrm{Mes}} \mathrm{PDP}{ }^{\mathrm{Ph}}\right) \mathrm{Fe}\left(\mathrm{N}_{4} \mathrm{Tol}_{2}\right)$ using a UKS5 approach. 